\title{
BRCA1 loses the ring but lords over resistance
}

\author{
Simon N. Powell
}

Memorial Sloan Kettering Cancer Center, Department of Radiation Oncology and Program in Molecular Biology, New York, New York, USA

\begin{abstract}
Germline breast cancer 1 (BRCA1) variants are associated with a high risk of breast and ovarian cancers. Many BRCA1-mediated cancers are initially responsive to platinum-based therapy; however, resistance commonly develops. The BRCA1 ${ }^{185 d e l A C}$ mutation is common in the Ashkenazi Jewish population and has been thought to result in loss of function due to the introduction of a stop codon in the $5^{\prime}$ region of the BRCA1 transcript. Two studies in this issue of the $J C I$ reveal that the BRCA185delAC mutation results in the production of BRCA1 that lacks the $\mathrm{N}$-terminal really interesting new gene (RING) domain. RING-less BRCA1 was shown to directly mediate chemoresistance, while maintaining some homologous recombination function. These results provide important insight into BRCA1 function and indicate that other truncated proteins could arise through similar alterations in codon usage.
\end{abstract}

\section{An unexpected product from} a 2-base pair deletion

Mutations in the tumor-suppressor gene breast cancer 1 (BRCA1) are associated with a variety of cancers. While many BRCA1-mutant cancers initially respond to platinum-based therapy, resistance commonly develops. Two articles published in this issue make the remarkable observation that a defect in the coding region of the N-terminal really interesting new gene (RING) domain of BRCA1 (such as the BRCA1 ${ }^{185 d e l A G}$ mutation, which is common in the Ashkenazi Jewish population) results in the utilization of an alternative start site for translation at the methionine at amino acid 297 (Figure 1). Normally, a 2-bp loss would result in a shift of the reading frame, and, ultimately, much of the transcript would not encode the amino acid sequence. Many of these transcripts are eliminated via nonsense-mediated decay (NMD) (1). Additionally, such a proximal frameshift would most likely result in a null allele (2). However, if transcription of such a mutant allele is unaffected, then initiation of translation at an alternative methio- nine is always possible. Indeed, given the results of these studies, it is possible that there are many truncated proteins, which are never observed or investigated, that arise by a similar mechanism. The smallersized protein is often assumed to be either a degradation product or a nonspecific cross-reactive band on an immunoblot that does not attract further attention.

Wang et al. observed that resistance to poly (ADP-ribose) polymerase inhibitors (PARPi) and cisplatinum developed in cells derived from a tumor of a BRCA1 $1^{185 d e l A C}$ mutation carrier (3). The dependency of BRCA1 function on BRCA1-associated RING domain 1 (BARD1) disappeared in the protein that was generated from this alternative start site of translation; therefore, in this context, BARD1 can be depleted without consequence. However, loss of BARD1 in WT BRCA1 results in BRCA1 dysfunction. The treatment resistance of $B R C A 1^{185 d e l A G_{-}}$-expressing tumor cells was reversed by depleting the BRCA1 message. Additionally, overexpression of a construct designed to generate the BRCA1 protein minus the N-terminal, BARD1-containing

\section{Related Articles: pp. 2903 and 3145}

domain resulted in chemoresistance in a previously sensitive cell line. Taken together, these observations support the view that translation from Met-297 of the BRCA1 site can produce a protein that is resistant to the DNA-damaging agents that normally make BRCA1-mutant cells sensitive.

The model for this chemoresistant effect conferred by the alternate protein is that there is no change in transcript size, but the secondary structure in the mutant mRNA is altered in a way that hinders other methionine residues in the N-terminal sequence, making Met-297 accessible to translation initiation. BRCA1 is known to have a number of alternative transcripts; however, this explanation was excluded by the data presented by Wang et al. (3), showing that the alternative transcripts are not abundantly expressed. Even though reexpressing BRCA1 from the Met-297 start site results in chemoresistance in a BRCA1deficient cell, this truncated protein is substantially less efficient than WT BRCA1 in homologous recombination (HR) function. The question that arises from these observations is: how does this alternative protein, which does not perform HR more efficiently than the full-length WT BRCA1 protein, result in resistance to the PARPi rucaparib and to cisplatin? The lack of a growth delay observed by Wang and colleagues when Met-297 BRCA1-expressing SUM1315 cells were grown as xenografts is remarkable.

A second article, by Drost et al. (4), provides complementary and additional information. Using genetically engineered mouse models that mimic the Ashkenazi Jewish BRCA1 mutations 185delAG and 5382 ins $C$, Drost and colleagues showed that tumors arising in the breast-specific Brca1 ${ }^{185 \text { stop }}$ mice express a RING-less protein, which mediates therapy resistance that is not seen in the equivalent Brca1 ${ }^{5382 s t o p}$ mice. This work from the Jonkers group at the Netherlands Cancer Institute (NKI) in Amsterdam extends the reports on the BRCA1 models they previously published and shows that breast-specific genetic alterations in a p53-deficient background result in breast tumor formation at a high 
A

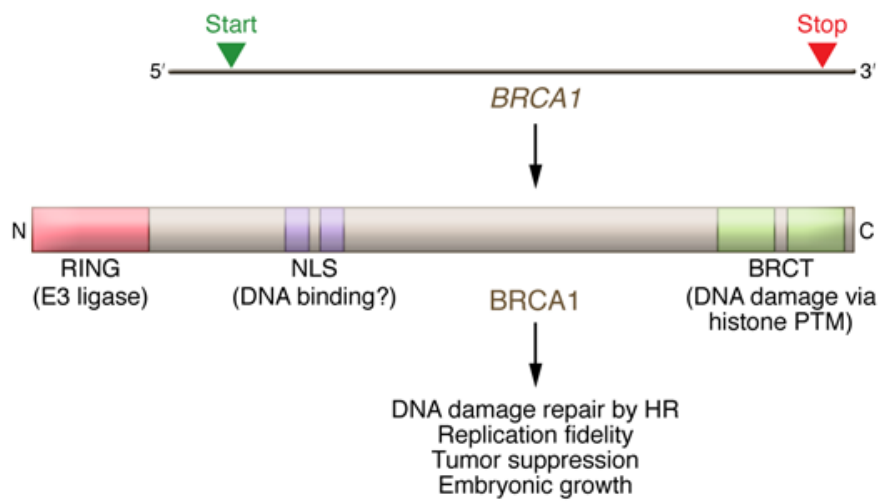

B

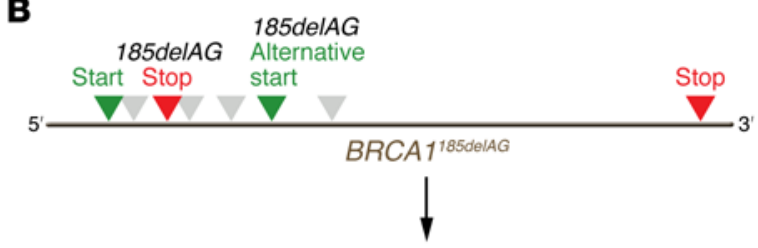

NLS BRCT

RING-less BRCA1

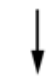

DNA damage repair by HR

Tumor drug resistance

Figure 1. Effects of the 185delAG mutation on BRCA1 function. (A) WT BRCA1 contains a RING domain (red), a nuclear localization sequence (NLS, purple) and a functional C-terminal domain (BRCT, green). The WT protein has multiple functions, including DNA damage repair, maintenance of replication fidelity, tumor suppression, and support of embryonic growth. PTM, posttranslational modification. (B) In this issue of the JCI, two studies demonstrate alternative translation of BRCA1 mRNA at the Met-297 codon (bright green arrow) in genetically defined human cells and genetically engineered mouse cells. The 185delAG mutation was thought to result in a frameshift mutation with an early stop codon (red arrow); however, this mutation supports translation at the Met-297 site, but not at other putative in-frame methionine residues (gray arrows). The alternative protein lacks the RING domain and does not prevent embryonic lethality or tumor formation; however, this truncated protein does allow DNA repair by HR at approximately $50 \%$ of the activity of WT BRCA1. One explanation may be the distinct roles of BRCA1 in DNA damage repair and replication fidelity. Alternative explanations are possible and include transcriptional changes via chromatin PTMs.

and predictable rate. It should be noted that constitutive expression of these alleles in embryonic stem cells results in embryonic lethality at E9.5, confirming that only WT BRCA1 can support normal development. The incidence of tumor formation in the mouse mammary gland with mammary-specific genetic alterations is almost identical, whether BRCA1 is absent or mutated (185stop and 5382stop). In addition, the chromosomal patterns of genomic instability (the large segment gains and losses found in BRCA-deficient tumors) are the same for all three mammary genotypes. At first, it appears that these stop alleles are behaving like null alleles of BRCA1 for tumor development and genomic instability. The crux of the findings of Drost and colleagues is that the 185stop allele and the resultant RING-less BRCA1 protein appears to retain a sufficient level of HR function (even though it is not quite as good as that of WT BRCA1) to result in resistance to olaparib PARPi and cisplatin. In contrast, this 185stop allele does not prevent the formation of tumors or allow embryonic development, suggesting that either the alternative RING-less BRCA1 protein is not expressed at early time points in embryonic or tumor development or that the RING-less BRCA1 protein cannot function in genomic integrity, whereas it can function in double-stranded break (DSB) repair by HR. The collective published data favor the latter explanation, as mice expressing the BRCA1-C61G mutation (5) also show resistance to HRdirected therapy but still develop tumors at a high rate. In contrast, the BRCA1-I26A mutant that lacks E3 ligase activity does suppress tumor formation in mice (6).

\section{Implications of RING-less BRCA1-mediated resistance}

The bigger-picture view of these data is quite provocative. First, there is the possibility that transcripts of genes with a frameshift do not always result in NMD, and if translation can initiate from a downstream methionine, hypomorphic alleles can be expressed that will modify the expected phenotype. The prevalence of this type of observation should be determined. Second, the role of the RING domain of BRCA1 continues to be puzzling, especially since the ability of these mutants to respond to DNA damage and promote HR is relatively well preserved in BRCA1 variants missing the entire RING domain. However, for genomic integrity and replication fidelity, the role of the RING domain may be much more important, because most disrupting alterations to the RING domain of BRCA1 result in embryonic lethality, genomic instability, and tumor development in targeted cells. Third, the DNA damage response of BRCA1 is very sensitive to WT BRCA1 gene dosage or the presence of hypomorphic alleles of BRCA1.

The question of which proteins are targeted by the E3 ligase activity of BRCA1 has been debated for many years (7). Initially, the resection initiation protein CTIP was thought to be a bone-fide target of BRCA1 (8), but maneuvers to impair the interaction of BRCA1 with CTIP do not phenocopy BRCA1 E3 ligase defects, suggesting that CTIP was an insufficient explanation of the findings. More recently, BRCA1 has been shown to ubiquitylate histones (H2A) (9), which might help to explain the rather extensive literature on the diverse effects of BRCA1 on DNA repair and transcription. In addition, the de-ubiquitylating enzyme BAP1, defined as a BRCA1-associated protein, but without direct effects on BRCA1-associated DNA repair (10), is also predominantly a histone-associated protein. The critical finding of the articles by Wang et al. (3) and Drost et al. (4) is that BRCA1, without the RING domain, can function fairly well in executing $\mathrm{HR}$ in response to DNA damage, but BRCA1 function at a level of genomic instability is much more dependent on the intact RING domain. Clearly, the signals and protein recruitment in response to exogenous DNA damage are significantly different 
from the signals and protein recruitment in response to naturally occurring replicative stress, which is the driver of genomic instability in most of these tumors.

BRCA1 and BRCA2 are thought to play a role in protection of the replication fork (11), which can be genetically dissociated from the DSB repair response. The best explanation of these findings is that RING-less BRCA1 maintains the capability for damage-induced HR, but lacks the capability to protect the replication fork from processes that would otherwise promote genomic instability. BRCA1 requires an intact C-terminal domain (BRCT domain) to be recruited to sites of DNA damage. For BRCA1 to promote resection of the 5 '-end, recent data support a role for the middle domain of BRCA1 to be intact $(12,13)$. Once the exogenous DNA damage is done, the protective role of BRCA1 requiring the RING domain is bypassed, and the recruitment and damage-processing roles of BRCA1 are all that are required (Figure 1).

\section{Conclusions and future directions}

In conclusion, these two articles identify potentially important properties of the BRCA1 protein and the mechanisms of therapy resistance. Some of the common tumor-associated germline mutations of BRCA1 that affect the RING domain, which have been associated with primary or acquired resistance to PARPi or platinum drugs, can now be explained by this mechanism of alternative translation. The cellular and mouse experiments both provide a consistent theme, in which stops in the RING domain allow alternative translation, but stops in the $\mathrm{C}$-terminal domain prevent recruitment to DNA damage- or replication-stressed chromatin and therefore affect all aspects of BRCA1 function. Future work can be done to support this model, by observing how BRCA1 is recruited to double-stranded DNA damage compared with blocked replication forks. The role of histone ubiquitylation cycling may also be critical to the replication-associated genomic instability observed in RING domain mutants of BRCA1. If it was not already clear, BRCA1 has been shown to be a truly complex pleiotropic protein involved in many aspects of genomic instability, histone marks, transcription, and many other reported functions. The alternative transcripts of BRCA1 may constitude another layer of activity that controls and dictates different roles for BRCA1 in cellular functions that has not yet been solved.

\section{Acknowledgments}

The author has received support from Memorial Sloan Kettering Cancer Center (MSKCC) P30 grant CA008748 and NIH grants R01 CA169306 and CA187069.

Address correspondence to: Simon Powell, 1275 York Avenue, New York, NY 10065, USA. Phone: 212.639.3639; E-mail: powells@mskcc.org.
1. Popp MW, Maquat LE. Leveraging rules of nonsense-mediated mRNA decay for genome engineering and personalized medicine. Cell. 2016;165(6):1319-1322.

2. Kurosaki T, Maquat LE. Nonsense-mediated mRNA decay in humans at a glance. JCell Sci. 2016;129(3):461-467.

3. Wang Y, et al. RING domain-deficient BRCA1 promotes PARP inhibitor and platinum resistance. J Clin Invest. 2016;126(8):3145-3157.

4. Drost $\mathrm{R}$, et al. $B R C A 1^{185 d e l A G}$ tumors may acquire therapy resistance through expression of RINGless BRCA1. J Clin Invest. 2016;126(8):2903-2918.

5. Drost R, et al. BRCA1 RING function is essential for tumor suppression but dispensable for therapy resistance. Cancer Cell. 2011;20(6):797-809.

6. Shakya R, et al. BRCA1 tumor suppression depends on BRCT phosphoprotein binding, but not its E3 ligase activity. Science. 2011;334(6055):525-528.

7. Barber LJ, Boulton SJ. BRCA1 ubiquitylation of CtIP: Just the tIP of the iceberg? DNA Repair (Amst). 2006;5(12):1499-1504.

8. Yu X, Fu S, Lai M, Baer R, Chen J. BRCA1 ubiquitinates its phosphorylation-dependent binding partner CtIP. Genes Dev. 2006;20(13):1721-1726.

9. Kalb R, Mallery DL, Larkin C, Huang JT, Hiom $\mathrm{K}$. BRCA1 is a histone-H2A-specific ubiquitin ligase. Cell Rep. 2014;8(4):999-1005.

10. Bott M, et al. The nuclear deubiquitinase BAP1 is commonly inactivated by somatic mutations and 3p21.1 losses in malignant pleural mesothelioma. Nat Genet. 2011;43(7):668-672.

11. Schlacher K, Wu H, Jasin M. A distinct replication fork protection pathway connects Fanconi anemia tumor suppressors to RAD51-BRCA1/2. Cancer Cell. 2012;22(1):106-116.

12. Tomimatsu N, et al. Phosphorylation of EXO1 by CDKs 1 and 2 regulates DNA end resection and repair pathway choice. Nat Commun. 2014;5:3561.

13. Paull TT, Cortez D, Bowers B, Elledge SJ, Gellert M. Direct DNA binding by Brca1. Proc Natl Acad Sci U S A. 2001;98(11):6086-6091. 